\title{
Research Article \\ Gaussian Curvature in Propagation Problems in Physics and Engineering
}

\author{
Ezzat G. Bakhoum \\ Department of Electrical and Computer Engineering, University of West Florida, \\ 11000 University Parkway, Pensacola, FL 32514, USA \\ Correspondence should be addressed to Ezzat G. Bakhoum, ebakhoum@uwf.edu
}

Received 1 September 2011; Accepted 9 October 2011

Academic Editor: Cristian Toma

Copyright (C) 2012 Ezzat G. Bakhoum. This is an open access article distributed under the Creative Commons Attribution License, which permits unrestricted use, distribution, and reproduction in any medium, provided the original work is properly cited.

The computation of the Gaussian curvature of a surface is a requirement in many propagation problems in physics and engineering. A formula is developed for the calculation of the Gaussian curvature by knowledge of two close geodesics on the surface, or alternatively from the projection (i.e., image) of such geodesics. The formula will be very useful for problems in general relativity, civil engineering, and robotic navigation.

\section{Introduction}

In many propagation problems in physics and engineering, it becomes necessary to compute the Gaussian curvature of a two-dimensional surface. In physics, this becomes necessary in the applications of general relativity, where it is sometimes desired to calculate the Gaussian curvature at a point in space from the observed geodesic paths of planets or light rays [1,2]. In engineering, engineers who are involved in the design of structures such as geodesic domes frequently require a practical formula for computing the Gaussian curvature, where relations exist between the Gaussian curvature at any point on the surface of the structure and the stability of such a structure [3]. In certain other engineering applications, such as computer vision and robotic navigation, engineers sometimes find themselves facing the complicated problem of having to compute the Gaussian curvature of a surface in order to calculate 3dimensional depth data (or range) [4-6].

From the basic principles of differential geometry, the Gaussian curvature $G$ at any point of a two-dimensional surface $S$ is given by

$$
G=k_{1} k_{2}
$$




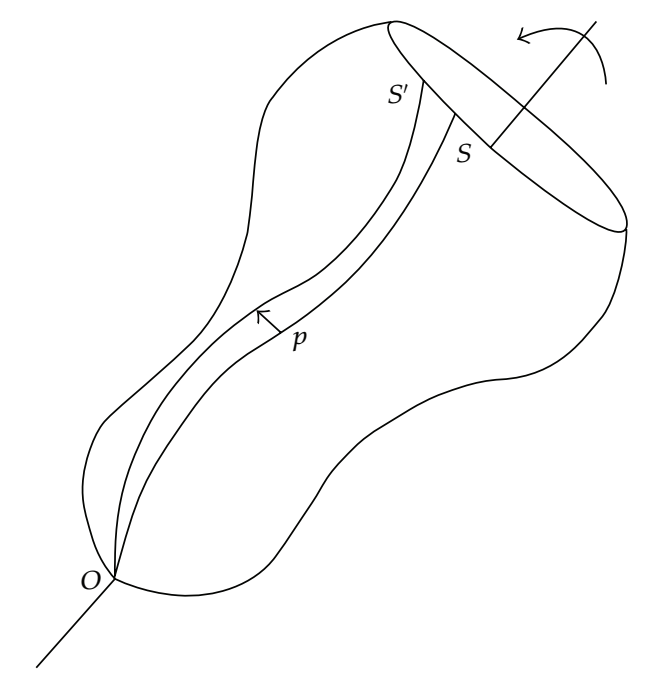

Figure 1: A general curve $S$ that is embedded in a surface of revolution and a copy $S^{\prime}$ that is separated from $S$ by a small rotation.

where $k_{1}$ and $k_{2}$ are the maximum and the minimum normal curvatures [6]. Unfortunately, in many practical situations, $k_{1}$ and $k_{2}$ are simply unknown. In the following section, we will derive a formula for computing the Gaussian curvature at any point on a surface by knowledge of two close geodesics on the surface, or alternatively from the projection (i.e., image) of such geodesics (this is very important in applications such as general relativity and robotic navigation, where no direct knowledge of the geodesics exists, but only an image of the geodesics is available). A simple test of the formula is given in Section 3 (the test shows that $G$, as computed from the formula, must vanish in an Euclidean 2-space). In Section 4, it is proven that the Gaussian curvature is a projective invariant and hence can be calculated from any projected image of two geodesics.

\section{Calculation of the Gaussian Curvature from Geodesic Deviation}

It is well known that any general 2-dimensional surface is topologically equivalent at any given point to a surface of revolution [6]. Hence, two close geodesics on the surface, when considered only within a small surface patch, can be treated as embedded in a surface of revolution. Such curves, however, will not necessarily be geodesics in the surface of revolution. Consider now a surface of revolution, where the smooth curve $S$ is a general curve that is embedded in the surface (Figure 1). $S^{\prime}$ is a copy of $S$ that is obtained by rotating $S$ through a small angle $\theta$.

$p^{r}$ is a position vector, defined over a circular ring passing by $S-S^{\prime}$. Let us select two parameters $u$ and $v$, such that $u$ varies as we travel along the curve $S$, but $v$ is constant, and $v$ varies as we pass from one curve to another, but $u$ is constant. Obviously,

$$
u=s, \quad v=\theta,
$$


where $\theta$ is the rotation angle of the axis from $S$ to $S^{\prime}$. Given such parameters on any surface in space, it can be shown that [7]

$$
\left(\nabla_{s \theta}^{2} \delta^{\rho}\right) d \theta-\left(\nabla_{\theta s}^{2} \delta^{\rho}\right) d \theta=\sum_{\zeta, \mu, \nu} R_{\zeta \mu \nu}^{\rho} \delta^{\zeta} \delta^{v} p^{\mu}
$$

where $\delta^{r}$ is the unit tangent vector to the curve, $R_{b c d}^{a}$ is the mixed curvature tensor [7], and the symbol $\nabla$ is the covariant derivative operator $[6,7]$. If the curve $S$ was a geodesic in the surface, we must have had $[6,7]$

$$
\nabla_{s} \delta^{r}=0
$$

since the covariant derivative of the unit tangent vector to a geodesic vanishes along the curve $[6,7]$. Since $S$ is a general curve, however, then $\nabla_{s} \delta^{r}$ will be the components of a vector of finite length, normal to the vector $\delta^{r}$ [6]. On the other hand, due to circular symmetry in a surface of revolution, the vector $\nabla_{s} \delta^{r}$, clearly, is parallel transported [6,7] along a circular ring in the surface. Hence, we must conclude that

$$
\nabla_{\theta}\left(\nabla_{s} \delta^{r}\right)=\nabla_{\theta s}^{2} \delta^{r}=0
$$

at any point on $S$. Further, given the parameters $s$ and $\theta$, it can be shown that [7]

$$
\left(\nabla_{s \theta}^{2} \delta^{r}\right) d \theta=\nabla_{s}^{2} p^{r}
$$

for any 2-dimensional surface. From (2.4) and (2.5), (2.2) is rewritten as

$$
\nabla_{s}^{2} p^{\rho}=\sum_{\zeta, \mu, v} R_{\zeta \mu \nu}^{\rho} \delta^{\zeta} \delta^{\mu} p^{v}
$$

where $\zeta, \mu, v=1,2$. Moreover, it has be shown that [7]

$$
R_{b c d}^{a}=G\left(\delta_{c}^{a} g_{b d}-\delta_{d}^{a} g_{b c}\right)
$$

for a smooth 2-dimensional manifold, where $G$ is the Gaussian curvature, $\delta_{b}^{a}$ is the Kronecker delta, and $g_{a b}$ are the components of the metric tensor at any point on the surface. Substituting from (2.7) into (2.6) and carrying out the summation, we obtain

$$
\nabla_{s}^{2} p^{\rho}+G p^{\rho}=G \delta^{\rho} \sum_{\mu, v} g_{\mu \nu} \delta^{\mu} p^{\nu}
$$

where we have used the identity

$$
\sum_{\mu, v} g_{\mu \nu} \delta^{\mu} \delta^{v}=1
$$




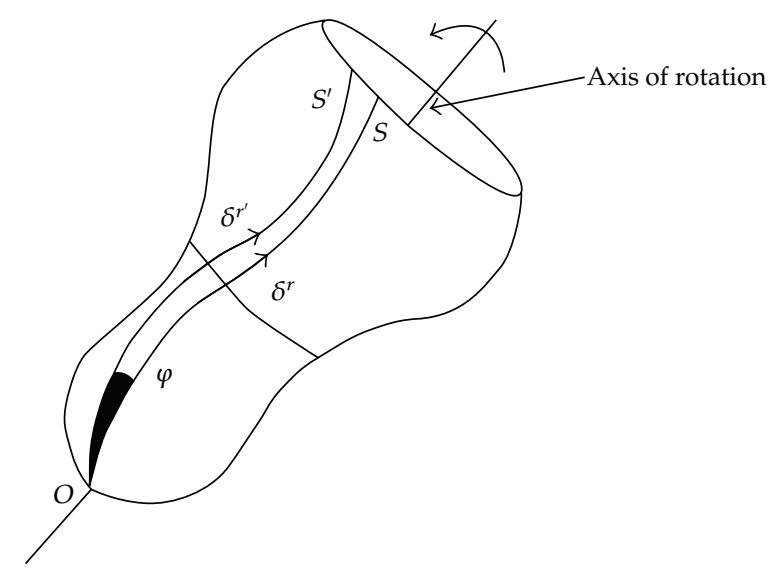

Figure 2: Unit tangent vectors to $S$ and $S^{\prime}$, respectively.

Equation (2.8) is analogous to the equation of geodesic deviation [6, 7]. Once again, if $S$ was a geodesic in the surface, we must have had an orthogonality condition

$$
\sum_{\mu, \nu} g_{\mu \nu} \delta^{\mu} p^{\nu}=0
$$

and (2.8) would have reduced to the well-known equation of deviation of two geodesics in a Riemannian 2-manifold. Equation (2.8), in its given form, will not allow the computation of the Gaussian curvature $G$, since the metric tensor components, as well as all the covariant derivatives on the surface, are unknown. However, (2.8) can be further reduced as follows: for an infinitesimal rotation $d \theta$,

$$
p^{r}=\frac{\partial x^{r}}{\partial \theta} d \theta
$$

Thus,

$$
\frac{\partial p^{r}}{\partial s}=\frac{\partial \delta^{r}}{\partial \theta} d \theta
$$

Now,

$$
\begin{aligned}
\delta_{S^{\prime}}^{r} & =\delta_{S}^{r}+\frac{\partial \delta_{S}^{r}}{\partial \theta} d \theta \\
& =\delta_{S}^{r}+\frac{\partial p^{r}}{\partial S}
\end{aligned}
$$

where $\delta_{S^{\prime}}^{r}, \delta_{S^{\prime}}^{r}$ are unit tangent vectors at $S$ and $S^{\prime}$, respectively, separated by a rotation $d \theta$ (Figure 2). 
Given that, for any vector $p^{r}$ on the surface [7],

$$
\nabla_{s} p^{\rho}=\frac{\partial p^{\rho}}{\partial s}+\sum_{\mu, \nu} \Gamma_{\mu \nu}^{\rho} p^{\mu} \frac{\partial x^{\nu}}{\partial s}
$$

where $\Gamma_{b c}^{a}$ is a Christoffel symbol of the second kind, we can always select coordinates such that Christoffel symbols vanish at the origin [6,7] (e.g., we can select coordinates on the surface, at the location of the vector $\left.p^{r}\right)$. Then, the vector $\sum_{\mu, \nu} \Gamma_{\mu \nu}^{\rho} p^{\mu} \delta^{\nu}$ is generally very small in the vicinity of the origin, and can be neglected (i.e., a linear approximation of $\nabla_{s} p^{\rho}$ is assumed here. This approximation will be further justified in the following discussion and in Section 3). Therefore, let

$$
\eta^{r}=\left(\delta_{S^{\prime}}^{r}-\delta_{S}^{r}\right)=\frac{\partial p^{r}}{\partial s} \approx \nabla_{s} p^{r}
$$

Further, let us define the deviation angle, $\psi$, as the angle between the two unit tangent vectors $\delta_{S^{\prime}}^{r} \delta_{S^{\prime}}^{r}$, at any point along the curve $S$. Generally, the angle between two curves is given by [7]

$$
\cos \psi=\sum_{\mu, \nu} g_{\mu \nu} \frac{d x^{\mu}}{d s} \cdot \frac{d x^{\nu}}{d s^{\prime}}
$$

but since $s=s^{\prime}$ is the length of the curve, and having $\delta^{r}=d x^{r} / d s$, we can write

$$
\cos \psi=\sum_{\mu, v} g_{\mu \nu}\left(\delta^{\mu}\right)_{S}\left(\delta^{v}\right)_{S^{\prime}}
$$

Hence, from (2.15) and (2.17),

$$
\begin{aligned}
\sum_{\mu, v} g_{\mu \nu} \eta^{\mu} \eta^{v} & =\sum_{\mu, v} g_{\mu \nu}\left[\delta_{S^{\prime}}^{\mu} \delta_{S^{\prime}}^{v}+\delta_{S}^{\mu} \delta_{S}^{v}-2 \delta_{S^{\prime}}^{\mu} \delta_{S}^{v}\right]=2(1-\cos \psi) \\
& \approx \sum_{\mu, v} g_{\mu \nu}\left(\nabla_{s} p^{\mu}\right)\left(\nabla_{S} p^{v}\right)
\end{aligned}
$$

We also see that

$$
\begin{aligned}
\cos \psi & \approx \sum_{\mu, \nu} g_{\mu \nu} \delta^{\mu}\left[\delta^{\nu}+\nabla_{s} p^{\nu}\right] \\
& \approx 1+\sum_{\mu, \nu} g_{\mu \nu} \delta^{\mu}\left(\nabla_{s} p^{\nu}\right) .
\end{aligned}
$$


Now, consider (2.8) and the summation

$$
\begin{aligned}
\sum_{\mu, \nu} g_{\mu \nu} p^{\mu}\left(\nabla_{s}^{2} p^{v}\right)+G \sum_{\mu, v} g_{\mu \nu} p^{\mu} p^{\nu} & =G \sum_{\alpha, \beta} g_{\alpha \beta} p^{\alpha} \delta^{\beta}\left[\sum_{\mu, v} g_{\mu \nu} \delta^{\mu} p^{\nu}\right] \\
& =G\left[\sum_{\mu, v} g_{\mu \nu} \delta^{\mu} p^{\nu}\right]^{2},
\end{aligned}
$$

and let

$$
P=\sqrt{\sum_{\mu, v} g_{\mu \nu} p^{\mu} p^{v}}
$$

denote the Euclidean norm of the vector $p^{r}$; thus,

$$
\begin{gathered}
\frac{d}{d s} P^{2}=2 \sum_{\mu, v} g_{\mu \nu} p^{\mu}\left(\nabla_{s} p^{v}\right) \\
\frac{d^{2}}{d s^{2}} P^{2}=2 \sum_{\mu, v} g_{\mu \nu}\left[p^{\mu}\left(\nabla_{s}^{2} p^{v}\right)+\left(\nabla_{s} p^{\mu}\right)\left(\nabla_{s} p^{v}\right)\right] .
\end{gathered}
$$

Substitution from (2.18), (2.21), and (2.23) into (2.20) gives

$$
\frac{1}{2} \frac{d^{2} P^{2}}{d s^{2}}-2(1-\cos \psi)+G P^{2}=G\left[\sum_{\mu, v} g_{\mu \nu} \delta^{\mu} p^{\nu}\right]^{2}
$$

To evaluate the last term in (2.24), we rewrite (2.8) as

$$
p^{\rho}=\delta^{\rho} \sum_{\mu, v} g_{\mu \nu} \delta^{\mu} p^{\nu}-\frac{1}{G} \nabla_{s}^{2} p^{\rho}
$$

Now, from (2.22) and (2.25), we have

$$
\begin{aligned}
\frac{d P^{2}}{d s}= & 2 \sum_{\mu, v} g_{\mu \nu}\left[\delta^{\mu} \sum_{\alpha, \beta} g_{\alpha \beta} p^{\alpha} \delta^{\beta}-\frac{1}{G} \nabla_{s}^{2} p^{\mu}\right]\left(\nabla_{s} p^{v}\right) \\
= & 2\left(\sum_{\mu, v} g_{\mu \nu} p^{\mu} \delta^{v}\right)\left(\sum_{\mu, v} g_{\mu \nu} \delta^{\mu}\left(\nabla_{s} p^{v}\right)\right) \\
& -\frac{2}{G} \sum_{\mu, v} g_{\mu \nu}\left(\nabla_{s}^{2} p^{\mu}\right)\left(\delta_{s^{\prime}}^{v}-\delta_{s}^{v}\right) .
\end{aligned}
$$


Each of the components in the last term of (2.26) vanishes identically. To prove this, we evaluate each of the components for each of the curves, $S$ and $S^{\prime}$, by substitution from (2.6). We have

$$
\begin{aligned}
\sum_{\mu, \nu} g_{\mu \nu} \delta^{\mu}\left(\nabla_{s}^{2} p^{\nu}\right) & =\sum_{\alpha} \delta^{\alpha}\left[\sum_{\rho, \zeta, \mu, \nu} g_{\alpha \rho} R_{\zeta \mu \nu}^{\rho} \delta^{\zeta} \delta^{\mu} p^{\nu}\right] \\
& =\sum_{\alpha, \zeta, \mu, \nu} R_{\alpha \zeta \mu \nu} \delta^{\alpha} \delta^{\zeta} \delta^{\mu} p^{\nu} \\
& =\sum_{\alpha, \zeta, \mu, \nu} G\left(g_{\alpha \mu} g_{\zeta \nu}-g_{\alpha \nu} g_{\zeta \mu}\right) \delta^{\alpha} \delta^{\zeta} \delta^{\mu} p^{\nu} .
\end{aligned}
$$

A straightforward summation shows that the right-hand side of (2.27) vanishes. We therefore conclude that $\nabla_{s}^{2} p^{r}$ is in the direction normal to the curve. In plus $\nabla_{s} p^{r}$ is in the direction of the tangent to the curve.

Finally, substitution from (2.19) into the first term of (2.26) gives

$$
\frac{d P^{2}}{d s}=2\left(\sum_{\mu, \nu} g_{\mu \nu} p^{\mu} \delta^{\nu}\right)(\cos \psi-1)
$$

or

$$
\sum_{\mu, \nu} g_{\mu \nu} p^{\mu} \delta^{v}=\frac{-d P^{2} / d s}{2(1-\cos \psi)}
$$

and hence (2.24) is further reduced to

$$
G=\frac{(1 / 2) d^{2} P^{2} / d s^{2}-2(1-\cos \psi)}{\left[\left(d P^{2} / d s\right) / 2(1-\cos \psi)\right]^{2}-P^{2}}
$$

where $G$ is the Gaussian curvature of the surface at the location of the vector $P$. In the following section, we prove that the Gaussian curvature given by (2.30) must vanish in an Euclidean 2-space. In Section 4, it is further proven that $G$ is a projective invariant and hence can be calculated from any projected image of the curves $S$ and $S^{\prime}$.

\section{Investigation of the Behavior of $G$ in an Euclidean Space}

Here, we illustrate by a simple example that the Gaussian curvature $G$, given by (2.30), must vanish in an Euclidean 2-space.

Consider a right circular cone, shown in Figure 3.

$P$ is the Euclidean norm of the position vector, and $\theta$ is the rotation angle (as discussed in the above text). 


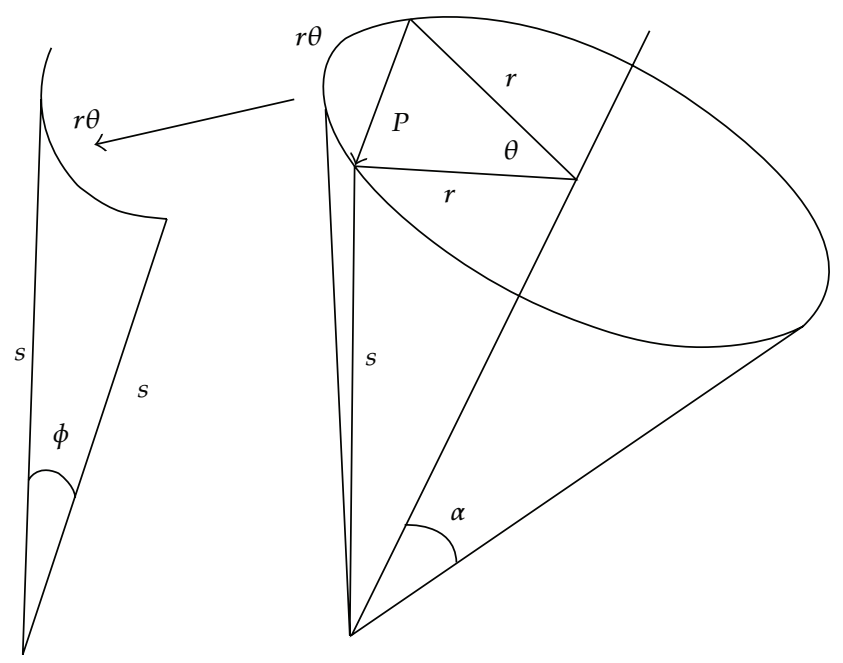

Figure 3: A right circular cone and the corresponding geometry.

From the Figure 3 , we see that

$$
\begin{gathered}
P^{2}=2 r^{2}(1-\cos \theta), \\
r=s \sin \alpha,
\end{gathered}
$$

where $s$ is the length of the generator. Hence,

$$
\begin{gathered}
P^{2}=2(1-\cos \theta)\left(s^{2} \sin ^{2} \alpha\right), \\
\frac{d P^{2}}{d s}=4 \sin ^{2} \alpha(1-\cos \theta) .
\end{gathered}
$$

Thus

$$
\frac{d^{2} P^{2}}{d s^{2}}=4 \sin ^{2} \alpha(1-\cos \theta)
$$

For an infinitesimal rotation, $\cos \theta$ is expressed by the first two terms of its power series, that is,

$$
\cos \theta \approx 1-\frac{\theta^{2}}{2 !}
$$

and thus, (3.3) is written as

$$
\frac{d^{2} P^{2}}{d s^{2}}=2 \sin ^{2} \alpha \theta^{2}
$$


Furthermore, we can see that

$$
r^{2} \theta^{2}=2 s^{2}(1-\cos \psi)
$$

where $\psi$ is the deviation angle, or

$$
(1-\cos \psi)=\frac{1}{2} \sin ^{2} \alpha \theta^{2}
$$

From (3.5) and (3.7), we have

$$
\frac{d^{2} P^{2}}{d s^{2}}=4(1-\cos \psi)
$$

By comparison of (2.30) and (3.8), we immediately see that $G$ must vanish in an Euclidean 2 -space. This proves the correctness of (2.30).

\section{Proof That the Gaussian Curvature G Is a Projective Invariant}

Now, we will reach our final goal by demonstrating that $G$, formulated by (2.30), can be measured directly in the image plane.

We rewrite (2.8) as

$$
G=-\frac{\nabla_{s}^{2} p^{\rho}}{p^{\rho}-\delta^{\rho} \sum_{\mu, \nu} g_{\mu \nu} \delta^{\mu} p^{\nu}}
$$

From (2.27), we saw that $\nabla_{s}^{2} p^{r}$ is a vector in the direction normal to the curve. Now, by using (2.9) and taking the summation

$$
\sum_{\mu, \nu} g_{\mu \nu} \delta^{\mu} p^{\nu}-\sum_{\mu, \nu} g_{\mu \nu} \delta^{\mu} \delta^{\nu}\left[\sum_{\mu, \nu} g_{\mu \nu} \delta^{\mu} p^{\nu}\right]=0
$$

it is easy to see that the denominator in (4.1) is also a vector in the direction normal to the curve.

If we now let

$$
\begin{gathered}
\nabla_{s}^{2} p^{\rho}=\alpha v^{\rho}, \\
p^{\rho}-\delta^{\rho} \sum_{\mu, \nu} g_{\mu \nu} \delta^{\mu} p^{v}=\beta v^{\rho},
\end{gathered}
$$

where $\alpha, \beta$ are scalars, and $v^{\rho}$ is a vector in the direction normal to the curve, then

$$
G=-\frac{\alpha}{\beta}
$$


Now, consider the orthographic projection of the two vectors in (4.3), written as

$$
\begin{aligned}
\overline{\nabla_{s}^{2} p^{\rho}} & =\sum_{\sigma} J_{\sigma}^{\rho}\left(\nabla_{s}^{2} p^{\sigma}\right)=\alpha \sum_{\sigma} J_{\sigma}^{\rho} v^{\sigma}, \\
\overline{p^{\rho}-\delta^{\rho} \sum_{\mu, \nu} g_{\mu \nu} \delta^{\mu} p^{\nu}} & =\sum_{\sigma} J_{\sigma}^{\rho}\left(p^{\sigma}-\delta^{\sigma} \sum_{\mu, \nu} g_{\mu \nu} \delta^{\mu} p^{\nu}\right) \\
& =\beta \sum_{\sigma} J_{\sigma}^{\rho} v^{\sigma},
\end{aligned}
$$

where $J_{b}^{a}$ is a transformation Jacobian between a coordinate system on the surface and a coordinate system in the image plane.

If measured in the image plane, the Gaussian curvature $G$ is now given by

$$
\bar{G}=-\frac{\overline{\nabla_{s}^{2} p^{\rho}}}{\overline{p^{\rho}-\delta^{\rho} \sum_{\mu, \nu} g_{\mu \nu} \delta^{\mu} p^{\nu}}}=-\frac{\alpha}{\beta^{\prime}}
$$

as we can easily see from (4.5).

Hence,

$$
G=\bar{G}
$$

The Gaussian curvature is therefore a projective invariant. It should be noted that, while orthographic projection is assumed, the image plane may be placed in any arbitrary position with respect to the curve $S$, and for all such positions, the Gaussian curvature $K$ holds the same numerical value. Equation (2.30) should be used in the image plane to obtain a correct measurement of $K$.

\section{References}

[1] S. K. Blau, “Gravity probe B concludes its 50-year quest,” Physics Today, vol. 64, no. 7, pp. 14-16, 2011.

[2] E. G. Bakhoum and C. Toma, "Relativistic short range phenomena and space-time aspects of pulse measurements," Mathematical Problems in Engineering, vol. 2008, Article ID 410156, 20 pages, 2008.

[3] T. Rothman, "Geodesics, domes, and spacetime," in Science a la Mode, Princeton University Press, 1989.

[4] R. O. Duda and P. E. Hart, Pattern Classification and Scene Analysis, Wiley, New York, NY, USA, 1973.

[5] B. K. Horn, Robot Vision, The MIT Press, 1987.

[6] M. P. do Carmo, Differential Geometry of Curves and Surfaces, Prentice-Hall, Englewood Cliffs, NJ, USA, 1976.

[7] J. L. Synge and A. Schild, Tensor Calculus, Dover, New York, NY, USA, 1978. 


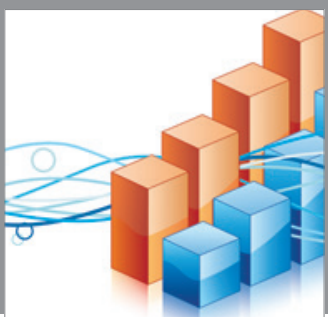

Advances in

Operations Research

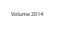

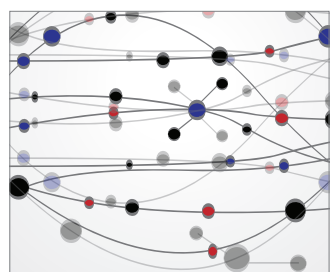

\section{The Scientific} World Journal
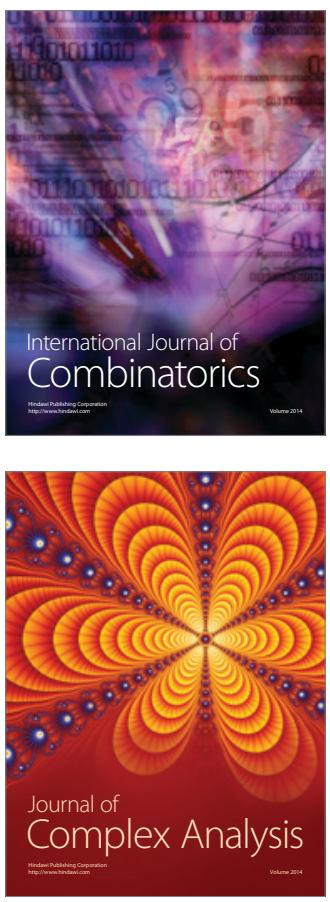

International Journal of

Mathematics and

Mathematical

Sciences
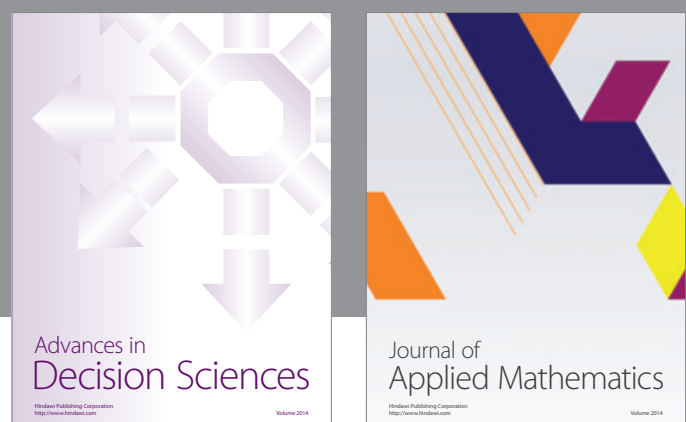

Journal of

Applied Mathematics
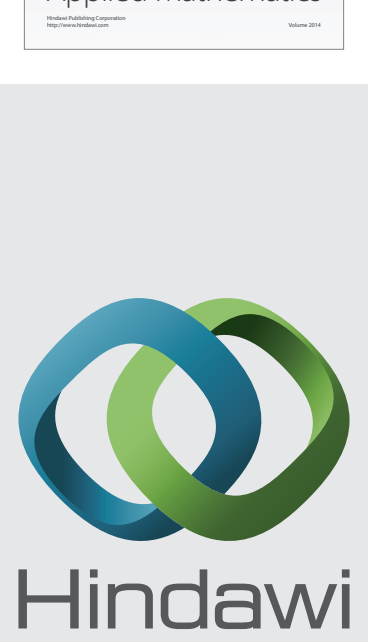

Submit your manuscripts at http://www.hindawi.com
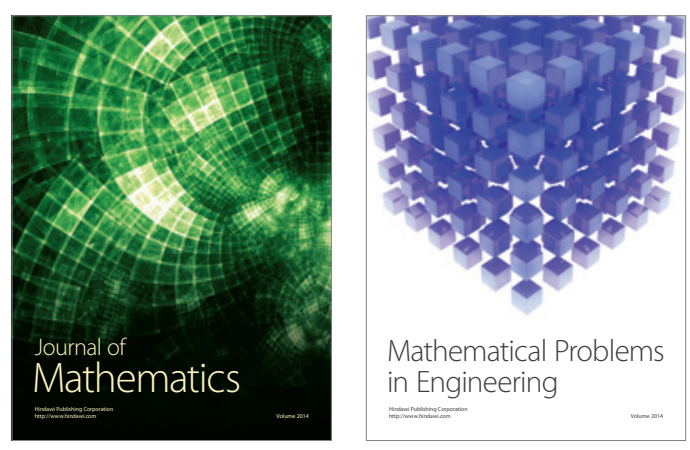

Mathematical Problems in Engineering
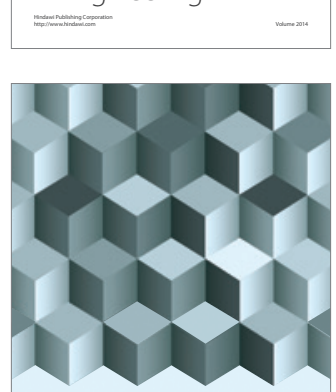

Journal of

Function Spaces
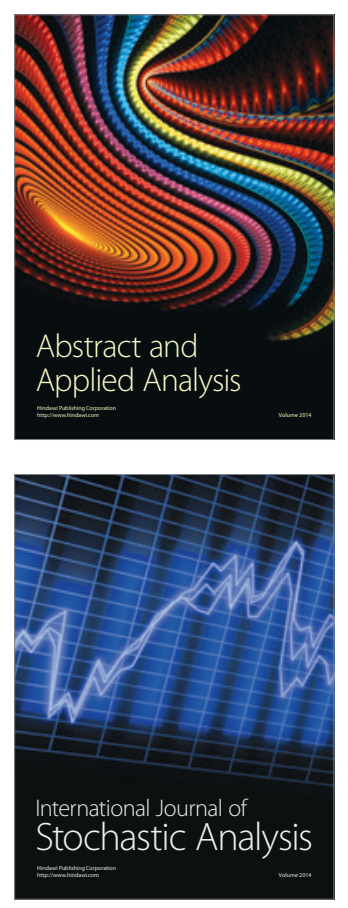

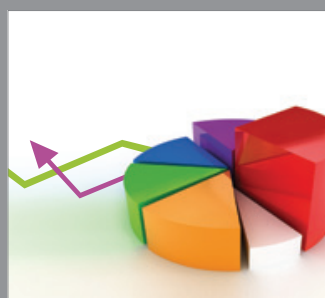

ournal of

Probability and Statistics

Promensencen
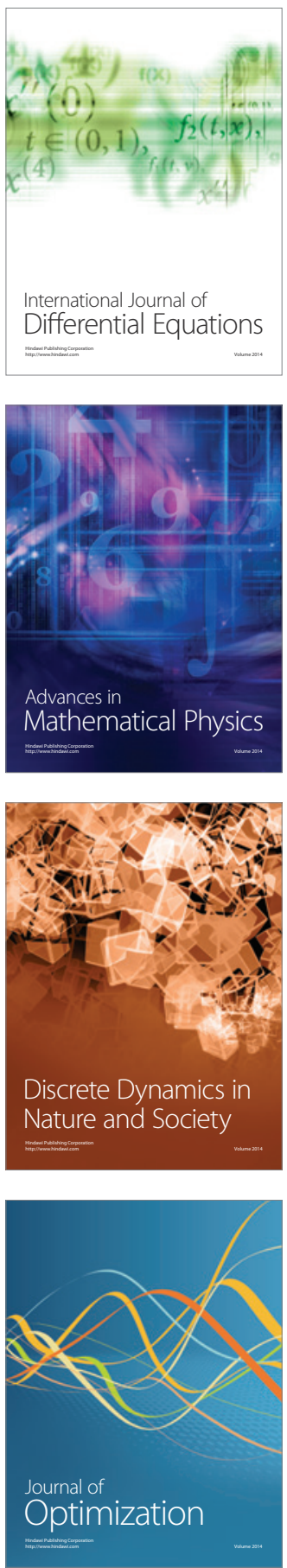